Voix et Images

voixetimages

\title{
Des nouvelles du printemps
}

\section{Martine-Emmanuelle Lapointe}

Volume 39, numéro 2 (116), hiver 2014

URI : https://id.erudit.org/iderudit/1025194ar

DOI : https://doi.org/10.7202/1025194ar

Aller au sommaire du numéro

Éditeur(s)

Université du Québec à Montréal

\section{ISSN}

0318-9201 (imprimé)

1705-933X (numérique)

Découvrir la revue

Citer ce compte rendu

Lapointe, M.-E. (2014). Compte rendu de [Des nouvelles du printemps]. Voix et Images, 39(2), 138-144. https://doi.org/10.7202/1025194ar d'utilisation que vous pouvez consulter en ligne.

https://apropos.erudit.org/fr/usagers/politique-dutilisation/ 


\author{
ROM A N \\ Des nouvelles du printemps \\ $+++$
}

MARTINE-EMMANUELLE LAPOINTE

Université de Montréal

Dans «Lettre à un étudiant ${ }^{1}$ ", une chronique qu'il a fait paraître en 2000, Michel Biron tentait de tirer quelques conclusions provisoires de ses lectures des romans contemporains. Plutôt pessimiste, il y affirmait notamment

[qu']écrire en 1965, c'était écrire dans la collectivité, en consonance avec l'ensemble des textes, des discours de la Révolution tranquille. En 2000, cette collectivité s'est fragmentée, les langages sociaux se sont atomisés, le romancier, lui, s'est professionnalisé, spécialisé: il écrit des romans pour les jeunes (ça marche bien, paraît-il), des fresques historiques, des romans populaires. Les gros romans ne manquent pas - phénomène relativement nouveau au Québec - , et ils vont désormais vers leur public comme la rivière se jette dans le fleuve. Quelques romanciers vivent ainsi de leur plume. Pourtant, jamais, depuis la Révolution tranquille, le roman québécois n'a-t-il été moins inspiré qu'actuellement. Une liste de noms ne serait pas utile; vous avez compris ce que je veux dire ${ }^{2}$.

Ces quelques phrases me sont revenues en tête dans le cadre d'un séminaire sur la littérature québécoise. Accompagnée d'étudiants d'une autre génération que celle du début des années 2000, je tentais assez malaisément d'y penser les rapports entre le littéraire et le politique à l'époque contemporaine, et plus particulièrement dans les nombreux textes qu'a inspirés la grève étudiante de 2012. Nous nous retrouvions malgré nous dans une sorte d'impasse, nous étions confrontés aux apories d'une réflexion condamnant à reconduire une conception sans doute désuète et naïve de l'engagement littéraire. Ce dernier se devait de reposer, nous semblait-il, sur une forme de croyance en un dogme, en un idéal, en un projet esthétique et idéologique ou, à l'inverse, sur une forme de refus de certains codes culturels et sociaux. En somme, l'écrivain engagé devait résister, refuser le prêt-à-penser, associé le plus souvent à la surconsommation, au néolibéralisme, à la culture du divertissement et, projet aussi

1 Michel Biron, «Lettre à un étudiant», Voix et Images, vol. XXV, nº 3, printemps 2000, p. 582-587. 2 Ibid., p. 583. 
vague que naï, proposer autre chose, créer autrement, faire «éclater les structures héritées ${ }^{3}$ ", comme le proposait jadis Hubert Aquin dans ses essais et ses romans. Circulaire, notre réflexion semblait toujours nous ramener aux mêmes interrogations sans cesse relancées : que serait concrètement cette manière de penser, d'écrire et de créer autrement? Quelles formes littéraires l'engagement contemporain pourrait-il emprunter? Pire, notre réflexion ranimait inlassablement les spectres d'une seule époque, ceux des grands écrivains de 1965... À côté des Aquin, Miron, Chamberland, Godin, à côté de ce panthéon très masculin, les auteurs des textes parus en marge du Printemps 2012 nous semblaient bien moroses. Le si beau vers de Miron scandé à maintes reprises pendant la grève étudiante, «Nous sommes arrivés à ce qui commence...», nous paraissait ainsi témoigner à la fois de l'instrumentalisation d'une conception figée de l'engagement littéraire et de son impossible dépassement.

Dans le cadre de cette chronique, je me propose de revenir sur les fictions du printemps étudiant, de même que sur Pourquoi Bologne ${ }^{4} \mathrm{~d}^{\prime}$ Alain Farah, afin d'esquisser quelques pistes de réflexion, encore bien embryonnaires, sur la question de l'engagement littéraire contemporain.

« Premier roman québécois inspiré de la grève étudiante de 2012 », précise la quatrième de couverture, Terre des cons ${ }^{5}$ de Patrick Nicol affiche rapidement ses couleurs. Dès le titre, nous sommes prévenus : le ton sera au pessimisme. À la Terre des hommes de l'Expo 67 succède la terre des cons de l'époque contemporaine, dont la banlieue dortoir du roman, proprette et tranquille, pourrait bien être l'emblème. Dans ce lieu sans histoire résonne encore l'écho de la grève étudiante de 2012, déjà révolue au moment du récit qui se déroule à l'été 2013. Politique, le roman l'est dans la mesure où il réfléchit sur les rapports entre la parole individuelle et la responsabilité collective, montrant bien que l'engagement ne va pas de soi à l'époque contemporaine, qu'il ressemble le plus souvent à un embrasement subit, sans suite, sans conséquences concrètes. Le narrateur, professeur de littérature dans un cégep régional, a embrassé la cause des étudiants sans trop y réfléchir, sans l'avoir choisie:

Quand la grève nous a rejoints, nous l'avons accueillie avec plaisir. Le printemps était
hâtif et nous avions besoin de repos. Nous avons toujours besoin de repos, c'est l'âge
ou la lourdeur de la tâche, je ne sais pas, mais à tous moments, nous pouvons nous
fermer les yeux et nous endormir sur place ou, préférablement, étendre nos jambes,
prendre un verre et nous convaincre que ce petit répit est amplement mérité. (53-54)

Si la grève étudiante a permis au narrateur de renouer momentanément avec les idéaux de sa jeunesse, elle n'a pu le délivrer de sa passivité bourgeoise. Le narrateur

3 Hubert Aquin, «Profession : écrivain», Point de fuite, Montréal, Cercle du livre de France, 1971, p. 58.4 Alain Farah, Pourquoi Bologne, Montréal, Le Quartanier, coll. «Série QR», 2013, 216 p. 5 Patrick Nicol, Terre des cons, Montréal, La Mèche, 2013, 98 p. 
est en effet un être d'habitudes. Il trouve le réconfort dans la bonne chère, dans la «musique sans voix» (31), dans «la ouate à laquelle [il a] tant aspiré» (25). Ses convictions se sont attiédies avec le temps. "Avant la grève, affirme-t-il, nous n'arrêtions pas de mesurer la profondeur du vide. » (52) Le vide est en lui, autour de lui. Sa maison, selon ses propres mots, est «un cube creux» (19). Par ennui ou par désœuvrement, il en vient à espionner ses jeunes voisins, s'introduisant la nuit dans leur appartement d'étudiants.

Ce n'est pas tant leur mode de vie qui fascine le narrateur, mais leur jeunesse. Dès l'incipit du roman, il évoque la perte de l'idéalisme qui semble inévitablement accompagner le passage des ans: « Je ne sais pas, Philippe, si en vieillissant on devient réac.» (9) La phrase, adressée au vieil ami, tout aussi bourgeois, épicurien et blasé que lui, hante le roman. Elle fait également écho au mal qui ronge le Chroniqueur - alter ego fictif de Richard Martineau -, une sorte de gangrène menaçant, de près ou de loin, tous ceux qui en vieillissant s'éloignent de ce qu'ils ont été: «Le Chroniqueur est en train de pourrir, j'en ai la conviction depuis longtemps. Un chancre s'est emparé de son corps, noircissant d'abord son gros orteil, qui s'est mis à puer. [...] Ça ne peut être le cancer, la gangrène ou quelque forme exotique du psoriasis, ce ne peut être que la mort.» (22-23) La mort de l'idéalisme, thème certes peu original, va ici de pair avec une réflexion sur la perte d'autorité de la parole littéraire. L'embourgeoisement conduirait à une forme de renoncement, de paresse culturelle. Non seulement le narrateur et ses proches lisent de moins en moins d'œuvres exigeantes, mais ils se réfugient dans le cynisme, choisissent la démission plutôt que l'action: «si les livres avaient un quelconque pouvoir, Madame Bovary aurait empêché Canal Vie» (48), affirme le professeur désillusionné.

Terre des cons, on l'aura compris, porte sur la fin des illusions. À la question «Es-tu heureux ?», posée plusieurs fois à Philippe, le narrateur ne saurait répondre, tant il est prisonnier des apparences trompeuses, du spectacle de son bonheur apparent. Dans sa vie sans histoires, la grève de 2012 constitue un moment de basculement qui a permis le dévoilement des véritables conflits et affrontements idéologiques. Sa fulgurance même a cependant frappé d'irréalité ce qu'elle a pu dévoiler. Sorte de brèche inattendue, elle a divisé le temps en un avant et un après. Elle a aussi brusquement renvoyé au silence les idéaux qu'elle avait réveillés, d'où sans doute le sentiment de vertige qui s'empare du narrateur près d'un an après sa conclusion.

Printemps spécial ${ }^{6}$ rassemble douze fictions rédigées par les romanciers de la maison d'édition Héliotrope. Inspirés de la grève étudiante de 2012, ces textes ont été écrits "dans la vibration aiguë du présent, dans son humilité », de préciser la quatrième de couverture. Inégaux, empruntant à divers styles et formes, ils témoignent éloquemment des problèmes rattachés à la fictionnalisation des événements politiques contemporains. Plusieurs des textes se présentent sous la forme de témoignages arti-

$$
++
$$

6 Collectif, Printemps spécial, Montréal, Héliotrope, coll. «Série K», 2012, 113 p. 
culés autour d'une parole subjective. Martine Delvaux se livre à la rédaction d'un «Autoportrait en militante» à la deuxième personne du singulier: "Tu écris debout au milieu des étudiants, des professeurs, des enfants, des grands-parents, des anticapitalistes, des féministes, des écologistes [...].» (29) Son texte se clôt sur une sorte d'épiphanie : «Tu n'es plus la même. Ton visage a changé. Voilà ce que tu vois. Le printemps t'a réinventée.» (33) Fidèle à sa pratique autofictionnelle, Gail Scott présente, dans "Chelsea rouge », le point de vue d'une écrivaine séjournant à New York pendant la grève de 2012. Cette dernière se mêle à la faune intellectuelle et artistique de la mégapole : «ici, on est engagés, militants, certains sont restés au Parc Zucchotti durant tout Occupy Wall Street» (57), précise-t-elle. À bonne distance des événements, dans une ville qui ne dort jamais et qui oublie vite, l'auteure se nourrit des menus faits de l'actualité, des discussions avec ses pairs, artistes, militants, étudiants, écologistes, semblables aux membres de la foule entourant le personnage de Delvaux. Deux des textes empruntent respectivement au journal intime et à la forme épistolaire, «Je n'étais pas là» d'André Marois et "Colère et tremblement» de Michèle Lesbre. «Atelier rouge» de Carole David tient davantage de l'histoire subjective des récentes réformes de l'enseignement collégial, considérées comme de "petites fins du monde » (75). Dans ce portrait peu reluisant, la grève de 2012 devient «l'éblouissement» même.

Éblouissement, épiphanie, moment de décrochage consacrant le retour à une parole engagée, la grève de 2012 se présente aussi comme le lieu d'épanouissement du citoyen engagé dans la vie de sa Cité. On ne témoigne que de soi, qu'à partir de soi, semblent soutenir plusieurs des fictions de Printemps spécial. Aurions-nous sans le savoir inversé la logique de la parole engagée des auteurs de 1965 qui écrivaient en chœur, en résonance avec les discours sociaux de leur époque, pour reprendre l'idée de Michel Biron? Au sein d'une foule constituée d'individus aux allégeances aussi nombreuses que diverses, le sujet vivrait une transfiguration. Son lyrisme personnel trahirait ainsi le type de rapport qu'entretiennent certains des auteurs - et leurs personnages - avec le politique: leurs discours n'est pas celui d'un "JE collectif ${ }^{7}$ " au sens où l'entendait Paul Chamberland en 1965, mais plutôt celui d'un NOUS atrophié ou d'un JE hyperbolique qui ne parviendrait jamais réellement à faire abstraction de son individualité, de sa singularité, de son appartenance à divers sous-groupes. Loin de moi l'intention de condamner cette conception des rapports entre l'individu et le collectif. Le «JE collectif», ne l'oublions pas, n'a fait qu'un temps, et n'a pas toujours donné lieu à des textes littéraires achevés. Il importe néanmoins de marquer l'écart entre le modèle de l'engagement littéraire hérité de la Révolution tranquille et ses avatars contemporains. L'unité du discours, de la parole, qui a toujours été une sorte de leurre commode, et parfois bon enfant, avouons-le, ne peut désormais constituer l'horizon de la pensée politique.

Sous son pire jour, l'engagement contemporain flirtera avec le narcissisme, confondra militantisme et carnaval, comme se plaît à le rappeler Catherine Mavrikakis dans son texte ouvertement ironique «À la casserole!». Mettant en scène un personnage prénommé Catherine, professeure d'université qui «ne fai[t] plus rêver les foules »

$$
+++
$$

7 Paul Chamberland, «Dire ce que je suis», Parti pris, n 5, janvier 1965, p. 39. 
(20) comme jadis, la nouvelle se joue des poncifs liés à l'engagement dans la sphère publique : «La grève étudiante avait donné un second souffle à Catherine... Elle avait l'impression que la morosité qu'elle avait connue dans les dernières années avait été balayée par le grand vent que portait en lui ce printemps québécois...» (19) «Second souffle ", "grand vent", «ce printemps québécois », la rhétorique repose ici sur des formules éculées connotant le recommencement, la renaissance, la promesse d'avenir. Grâce à la grève, Catherine «retrouv[e] l'amour de ses étudiants» (21) et l'espoir en une génération qui redécouvre les écrits d'Angela Davis et la Bande à Baader. Mais le ciel s'assombrit le jour où elle croise Hervé, étudiant brillant qu'elle a connu à l'époque de ses études en lettres. Hervé a décroché, est devenu itinérant. Personnage à peine esquissé, fantôme d'une autre époque, il fait écho aux Hervé emportés par le sida de Deuils cannibales et mélancoliques ${ }^{8}$ et renvoie momentanément Catherine à ses leurres, à ses illusions, à ses mesquineries. Mais la lucidité n'est que passagère. Bien vite Catherine retourne à son aveuglement, se disant qu'«après tout ce n'était peut-être pas son Hervé... Celui-ci était peut-être mort du sida dans les années 1990 ou encore était devenu attaché politique au Parti libéral... Qu'en savait-elle?» (25)

Pourquoi Bologne d'Alain Farah n'est pas à proprement parler un roman politique. Il ne traite pas directement de la question de l'engagement à l'époque contemporaine. La grève étudiante de 2012 y est rapidement évoquée et n'y apparaît pas comme un événement particulièrement signifiant. Voyageant dans les canaux d'interconnexion qui courent sous le campus de McGill, le narrateur se retrouve soudain mêlé à la manifestation «Occupy McGill» tenue le 10 novembre 2011, l'un des préludes aux événements du Printemps 2012. Entre cette brève séquence et les autres événements qui traversent le roman, il ne faudrait pas chercher à esquisser des liens trop étroits. Il serait même incongru de tenter de reconstruire de manière linéaire la trame d'un roman qui se moque ouvertement de la notion de cohérence. Empruntant à l'autofiction, au roman d'espionnage et à la science-fiction, Pourquoi Bologne met en scène un narrateur appelé Alain Farah, professeur à l'Université McGill, qui vit simultanément en 1962 et en 2012. Il engage une jeune femme, prénommée Candice, pour l'aider à rédiger son roman. Sur les conseils de celle-ci, il projette de consulter le $\mathrm{D}^{\mathrm{r}}$ Penfield pour chasser ses lubies et ses hallucinations. Au dernier moment, il se rend plutôt au Allan Memorial, où il tombe dans les griffes du vil $\mathrm{D}^{\mathrm{r}}$ Cameron. Ce dernier offre au narrateur l'occasion d'un étonnant voyage dans sa mémoire familiale ainsi que dans les citernes du bassin McGill. Voilà pour le résumé, partiel et peu fidèle à la riche matière du roman. Pour y être fidèle, il aurait fallu y traiter aussi des motifs obsédants, des liens entre la littérature et la science, entre McGill et la CIA, de la réflexion sur l'écriture autobiographique, du retour au roman familial, de la fascination pour les dessous de l'Histoire et des histoires, de la présence des spectres tutélaires d'Hubert Aquin et d'Umberto Eco... Je n'y parviendrai pas, autant vous prévenir.

$$
+++
$$

8 Catherine Mavrikakis, Deuils cannibales et mélancoliques, Montréal, Éditions Trois, 2000, 216 p. 
Le roman cultive volontiers l'anachronisme, se plaît à multiplier les références à la littérature consacrée et à la culture populaire. Surtout, il brouille les frontières entre réalité historique et fiction. S'il renvoie à une personne bien vivante, à l'auteur Alain Farah, le narrateur n'en demeure pas moins un être de fiction. Si le $\mathrm{D}^{\mathrm{r}}$ Cameron a bel et bien œuvré au Allan Memorial Institute dans les années 1950-1960, menant des expériences de déprogrammation sur ses patients, si McGill entretenait probablement des relations troubles avec la CIA, la fiction l'emporte. Tout est vrai et tout est faux dans Pourquoi Bologne: «Je n'existe pas» (84), "tout est possible ici» (66), affirme le narrateur afin d'invalider toute forme de théorie du reflet. Alain Farah, l'auteur et/ou le narrateur, va encore plus loin : il procède à des renversements, la fiction devenant plus réelle que la vie; il abolit les frontières entre les œuvres, se promenant d'un texte à l'autre, d'un film à l'autre sans égard pour la vraisemblance ou la juste référence. En voici un bref exemple:

\begin{abstract}
J'ai souvent en tête, aux aurores, cette phrase d'un vieux professeur d'histoire de l'art que je l'entends encore nous dire, debout devant l'écran sur lequel il projette des diapositives de tableaux de Géricault et du Caravage: «Il y a des peintres de la nuit, comme Rembrandt ou De La Tour. Mais il y a très peu de peintres de l'aube, de peintres de la lumière glauque.» (67)
\end{abstract}

Le lecteur aura reconnu ou non un passage du film Le déclin de l'empire américain de Denys Arcand. Incarné par le comédien Yves Jacques, le professeur d'histoire de l'art - pas si âgé d'ailleurs - tient en effet ces propos, à quelques détails près ${ }^{9}$. Le souvenir du narrateur - mais s'agit-il du souvenir d'une expérience vécue ou d'une simple réminiscence cinématographique? - est emprunté à la fiction d'Arcand, laquelle s'intègre à la vaste bibliothèque explorée par Alain Farah. L'intertextualité est ainsi au fondement même de l'écriture de Pourquoi Bologne. L'auteur pratique l'autocitation en évoquant son premier roman, Matamore no 29, ou l'un des articles qu'il a fait paraître dans Le Devoir. Le procédé renvoie à une conception de l'écriture et de la littérature qui n'est pas sans rappeler les théories aquiniennes de l'originalité littéraire. L'écrivain de Pourquoi Bologne est condamné à la répétition, ne pouvant livrer qu'une "variante désordonnée d'autres livres écrits par des écrivains inconnus ${ }^{10}$ ", comme l'affirmait le narrateur de Prochain épisode.

Le spectre d'Aquin hante Pourquoi Bologne, en informe l'écriture et la trame. Effets d'écho, correspondances multiples, «miroirs langagiers, culturels, idéologiques, [...] disposés comme des pièges ${ }^{11}$ » se multiplient : un certain Hamadou Diop (22, 25, 69, 95-96, 100), et non Hamidou comme dans Prochain épisode, s'amuse à piéger le narrateur. La Suisse, les voitures, les manoirs lugubres, les emprunts au roman

$$
+++
$$

9 La citation exacte se lirait comme suit: «Il y a des peintres de la nuit, comme Rembrandt ou Georges De La Tour. Mais il y a très peu de peintres de l'aube, parce que l'aube c'est l'heure de la mort, l'heure de la lumière glauque », Denys Arcand, Le déclin de l'empire américain, 1986. 10 Hubert Aquin, Prochain épisode, Montréal, Bibliothèque québécoise, 1995 [1965], p. 86. 11 René Lapierre, L'imaginaire captif, Montréal, Quinze, coll. «Prose exacte», 1981, p. 14 
d'espionnage et les clins d'œil à l'œuvre d'Umberto Eco, la présence de l'Institut Albert-Prévost où Aquin fut interné, la consommation de psychotropes, les théories sur le plagiat littéraire, toutes ces références ramènent de près ou de loin aux obsessions aquiniennes. Si Aquin a "laiss[é] les vrais romans aux vrais romanciers ${ }^{12}$ ", Farah se contente de «laisser les romans aux romanciers» (195). Manière de dire de nouveau qu'il renonce lui aussi à l'authenticité et à la vérité romanesques. J'en reviens à la question du politique. L'œuvre d'Hubert Aquin ne devrait-elle pas ressusciter les embrasements idéologiques de la Révolution tranquille, les réflexions sur l'aliénation et sur les rapports entre dominé et dominant? La dimension politique de l'écriture aquinienne est peu travaillée dans Pourquoi Bologne. C'est à l'autre versant de l'œuvre, à sa part testimoniale et mémorielle, que s'attache Alain Farah. La folie et la fragmentation identitaire, voire l'aliénation individuelle et non collective, sont au cœur du roman et rappellent cette leçon tout aquinienne : «le moi est un intertexte ${ }^{13}$ ».

Au début de Pourquoi Bologne, le narrateur raconte qu'il a vu le film Les ordres de Michel Brault au cinéma Palace en 1975. Il en évoque la séquence suivante:

- Je m'appelle Jean Lapointe et, dans le film, je suis Clermont Boudreau. Je suis né sur une ferme, Marie aussi. J'ai bien l'impression que notre plus grosse erreur, ça a été de nous en venir en ville. Là, je suis dans le textile, pis notre espoir, c'est notre syndicat.

Je pense souvent à cet instant où l'acteur devient le personnage, et je me demande si ce passage d'un mode à l'autre, Jean Lapointe le sent physiquement.

Moi, je m'appelle Alain Farah et, dans le livre, je suis Alain Farah. (26)

Je vais peut-être aller trop loin (mais n'est-ce pas ce que souhaite l'auteur?) en conférant une portée plus générale au passage d'un mode à l'autre. S'il est un moment particulièrement politique du roman, voire emblématique d'un certain rapport à l'Histoire collective, ce serait peut-être celui-là. Le film à teneur politique, consacré aux récits des victimes de la crise d'Octobre, est ici détourné. Jean Lapointe avait pour mission d'endosser le rôle du témoin, il parlait au nom des autres, des silencieux, des bafoués de l'Histoire. Alain Farah parle en son nom sans trop savoir ce qui lui revient en propre. Il est à la fois lui-même et autre, mais ne saurait dire de quoi il témoigne précisément... L'Histoire ne lui appartient plus, elle s'est défilée, télescopée, reposant sur de multiples versions des faits, vraies et fausses à la fois.

12 Hubert Aquin, Prochain épisode, p. 12. 13 Hubert Aquin, «Le texte ou le silence marginal ?», Blocs erratiques, Montréal, Quinze, coll. «10/10», 1982, p. 271. 